\title{
Heparin-binding epidermal growth factor contributes to COPD disease severity by modulating airway fibrosis and pulmonary epithelial-mesenchymal transition
}

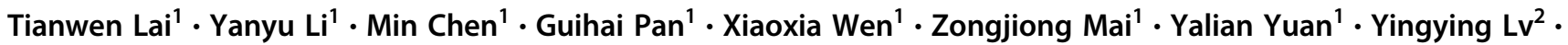 \\ Quanchao $\mathrm{Lv}^{1} \cdot$ Ruilian $\mathrm{Cen}^{3} \cdot$ Huajuan $\mathrm{Yi}^{1} \cdot$ Mengling $\mathrm{Wen}^{2} \cdot$ Dongming $\mathrm{Li}^{1} \cdot \mathrm{Chao} \mathrm{Cao}^{4} \cdot$ Dong $\mathrm{Wu}^{1} \cdot \mathrm{Bin} \mathrm{Wu}^{1}$
}

Received: 10 August 2017 / Revised: 30 January 2018 / Accepted: 6 February 2018 / Published online: 26 March 2018

(c) United States \& Canadian Academy of Pathology 2018

\begin{abstract}
Although airway fibrosis and epithelial-mesenchymal transition (EMT) contribute to airway remodeling in chronic obstructive pulmonary disease (COPD), the mechanisms underlying their development have not been fully elucidated. In the present study, we aimed to assess heparin-binding epidermal growth factor (HB-EGF) expression in the airways of patients with COPD and to elucidate the possible role of HB-EGF in the pathology of COPD. Sputum and lung tissue HB-EGF expression was evaluated in control subjects and patients with COPD. The relationships between HB-EGF expression, disease severity, collagen deposition (fibrosis), and EMT were investigated. In vitro, human bronchial epithelial (HBE) cells and lung fibroblast cells exposed to the recombinant HB-EGF, collagen deposition and EMT were assessed. We found that sputum HB-EGF expression was significantly increased in patients with COPD compared with non-smokers and smokers without COPD. There was a significant positive correlation between sputum HB-EGF and COPD assessment test (CAT) score. HB-EGF expression was significantly increased in the lung tissue samples of patients with COPD and associated with collagen deposition and $\mathrm{N}$ - and E-cadherin, and vimentin expression. In vitro, HB-EGF promoted collagen production in lung fibroblasts. Moreover, HB-EGF induced the EMT process through induction of $\mathrm{N}$-and E-cadherin, and vimentin expression in HBE cells. Collectively, HB-EGF induces airway remodeling by modulating airway fibrosis and pulmonary EMT, and contributes to the COPD severity. The current data may provide insight into the underlying pathogenesis of COPD, in which HB-EGF has an important pathogenic role.
\end{abstract}

\section{Introduction}

Chronic obstructive pulmonary disease (COPD) is a chronic inflammatory respiratory condition, characterized by

These authors contributed equally: Tianwen Lai, Yanyu Li, Min Chen.

Electronic supplementary material The online version of this article (https://doi.org/10.1038/s41374-018-0049-0) contains supplementary material, which is available to authorized users.

$\triangle$ Bin Wu
wubin621011@126.com
$\square$ Dong Wu
wudong98@126.com
$\square$ Chao Cao
caocdoctor@163.com

1 Department of Respiratory and Critical Care Medicine, The progressive airflow limitation that is not fully reversible [1]. Airway fibrosis and epithelial-mesenchymal transition (EMT) contribute to airflow limitation and resistant to current therapies, including corticosteroids [2,3]. Despite advances in understanding the inflammatory features of COPD, the mechanisms underlying the airway fibrosis and EMT remain poorly understood.

Heparin-binding epidermal growth factor (HB-EGF) is a member of the epidermal growth factor (EGF) superfamily [4]. HB-EGF is a potent chemotactic factor for epithelial,

Affiliated Hospital of Guangdong Medical University, Zhanjiang 524001, China

2 Department of Rehabilitation, The Affiliated Hospital of Guangdong Medical University, Zhanjiang 524001, China

3 Department of Rehabilitation, Yangjiang People's Hospital, Yangjiang 529500, China

4 Department of Respiratory Medicine, Ningbo First Hospital, Ningbo 315010, China 
smooth muscle, and mesenchymal stem cells [4, 5]. Wang et al. [6] reported the level of HB-EGF expression correlated to airway smooth muscle (ASM) mass thickening, and that it induced ASM migration and proliferation in vitro. Hassan et al. [7] showed that elevated HB-EGF expression within the asthmatic ASM mass occurred. However, information is still lacking regarding whether HB-EGF occurs at an early stage of disease or accelerates COPD disease progression.

The airway epithelium assures frontline innate defense mechanisms via a physical barrier and secretion of protective factors. Epithelial-mesenchymal transition (EMT) is a process through which epithelial cells that normally interact with basement membranes via their basal surface undergo multiple biochemical changes $[8,9]$. EMT is involved in bronchial remodeling and loss of lung function [10]. Peribronchial and subepithelial fibrosis in peripheral airways are major causes for airway remodeling in COPD and are responsible for the airflow limitation. It has been previously shown that increased extracellular matrix (ECM) deposition is a key structural change of the remodeled airway wall and drives the cellular processes of proliferation, migration, repair, and inflammation [2, 3, 11]. Among lung resident cells, fibroblasts play a key role in tissue remodeling and fibrosis. Fibroblast-mediated airway fibrosis and ECM protein deposition play an important role in peripheral airway narrowing in COPD [3, 11]. However, whether HBEGF participates in EMT's functional consequences and fibrosis in the COPD patients' conducting airways remains unclear.
Based on this evidence, we designed the present study to determine three points: (1) whether the levels of sputum HB-EGF are elevated in COPD patients' airways and to identify its correlations to clinical parameters (such as lung function and disease severity); (2) whether HB-EGF expression is upregulated in COPD patients' lung tissues and to identify its correlations to airway fibrosis and EMT; and (3) human lung fibroblasts and bronchial epithelial (HBE) cells were stimulated by HB-EGF in order to analyze collagen deposition and EMT.

\section{Materials and methods}

\section{Patient selection and clinical data}

To investigate the levels of HB-EGF in the airways, a total of 35 smokers with stable COPD as defined by the Global Initiative for Chronic Obstructive Lung Disease (GOLD) guidelines [12], who had a postbronchodilator forced expiratory volume in $1 \mathrm{~s}\left(\mathrm{FEV}_{1}\right)$ /forced vital capacity (FVC) ratio $<0.7$ were recruited for the induced sputum study after receiving written informed consent. Patients didn't receive oral or intravenous corticosteroids or any other antiinflammatory drugs during the preceding 4 weeks. Stable COPD was defined as no change in patients' treatment course for four or more weeks with no evident acute COPD exacerbation during that same time period [12]. We also recruited 20 age-matched non-smokers with normal spirometry and 26 smokers with normal spirometry (as controls)

Table 1 Characteristics of the subjects in the sputum study

\begin{tabular}{lccc}
\hline Parameters & $\begin{array}{c}\text { Non-smokers without } \\
\text { COPD }\end{array}$ & $\begin{array}{c}\text { Smokers without } \\
\text { COPD }\end{array}$ & $\begin{array}{c}\text { Smokers with } \\
\text { COPD }\end{array}$ \\
\hline Age, yrs & $64.5 \pm 1.8$ & $63.7 \pm 1.1$ & $64.2 \pm 1.4$ \\
Female/male, $n$ & $8 / 12$ & $10 / 16$ & $15 / 20$ \\
Smokers, former/current, & NA & $13 / 13$ & $16 / 19$ \\
$n$ & & & 0.931 \\
FEV,,$L$ & $2.40 \pm 0.13$ & $2.29 \pm 0.11$ & $1.39 \pm 0.10$ \\
FEV1, \%Pred & $104.6 \pm 4.10$ & $97.48 \pm 3.94$ & $52.91 \pm 3.01$ \\
FEV $/$ FVC, $\%$ & $82.72 \pm 1.10$ & $80.26 \pm 1.14$ & $52.24 \pm 1.58$ \\
GOLD I-II/III-IV, $n$ & NA & NA & $18 / 17$ \\
CAT Scores & NA & NA & $22.4 \pm 1.0$ \\
Treatment regimens, $n$ & NA & NA & $<0.001$ \\
ICS/LABA & & & - \\
LAMA & & & 25 \\
SABA & & & 7 \\
Theophylline & & & 15 \\
\hline
\end{tabular}

Data are presented as mean \pm SEM, unless otherwise stated

$B M I$ body mass index, $C A T$ COPD assess test, COPD chronic obstructive pulmonary disease, $F E V_{l}$ forced expiratory volume in $1 \mathrm{~s}, F V C$ forced vital capacity, GOLD The Global Initiative for Chronic Obstructive Lung Disease, LABA long-acting $\beta$ agonist, LAMA long-acting muscarinic agonist, $N A$ not applicable, SABA short-acting $\beta 2$ agonist 
Table 2 Characteristics of the subjects in the lung tissue study

\begin{tabular}{|c|c|c|c|c|}
\hline Parameters & $\begin{array}{l}\text { Non-smokers without } \\
\text { COPD }(n=9)\end{array}$ & $\begin{array}{l}\text { Smokers without } \\
\text { COPD }(n=10)\end{array}$ & $\begin{array}{c}\text { Smokers with } \\
\text { COPD }(n=30)\end{array}$ & $p$ value \\
\hline Female/male, $n$ & $3 / 6$ & $3 / 7$ & $11 / 19$ & 0.925 \\
\hline Age, yrs & $61.8 \pm 3.7$ & $63.8 \pm 3.5$ & $61.7 \pm 1.2$ & 0.790 \\
\hline $\begin{array}{l}\text { Smokers, former/current, } \\
n\end{array}$ & & $5 / 5$ & $9 / 21$ & 0.251 \\
\hline $\mathrm{FEV}_{1}, \%$ Predicted & $94.7 \pm 2.9$ & $84.8 \pm 2.7$ & $49.5 \pm 1.9$ & $<0.001$ \\
\hline $\mathrm{FEV}_{1} / \mathrm{FVC}, \%$ & $78.9 \pm 1.5$ & $75.3 \pm 1.1$ & $53.0 \pm 1.8$ & $<0.001$ \\
\hline GOLD I-II/III-IV, $n$ & NA & NA & $12 / 18$ & NA \\
\hline \multicolumn{5}{|l|}{ Cancer type, $n$} \\
\hline Squamous cell carcinoma & 2 & 7 & 19 & 0.226 \\
\hline Adenocarcinoma & 5 & 2 & 8 & \\
\hline Small-cell lung cancer & 2 & 1 & 3 & \\
\hline
\end{tabular}

Values are mean \pm SEM, unless otherwise stated

$C O P D$ chronic obstructive pulmonary disease, $F E V_{l}$ forced expiratory volume in $1, F V C$ forced vital capacity, $N A$ not applicable

from the communities surrounding our hospital. They were free of respiratory tract infection during. the 4 weeks prior to the study. Patients' and controls' characteristics are shown in Table 1.

To investigate the expression of HB-EGF in the lung tissue and identify its correlation to airway epithelial-mesenchymal transition (EMT) and fibrosis, lung tissue specimens were obtained from the subpleural parenchyma of the lobe that was resected at surgery. Specimens were dissected at a distance of $\geq 5 \mathrm{~cm}$ away from the tumor. Patients who had respiratory infections or received oral or intravenous corticosteroids during the preceding 4 weeks, a previous history of other cancer, and had undergone radiotherapy or chemotherapy were excluded. A total of nine non-smokers with normal spirometry, 10 smokers without COPD, and 30 smokers with COPD were enrolled. Twelve GOLD I/II patients and 18 GOLD III/IV patients had undergone lung cancer surgery. Patients' characteristics are shown in Table 2. Primary human lung fibroblasts were isolated from lung parenchyma from a subgroup $(n=4)$ of these subjects.

The corresponding protocol was approved by the Ethics of Research Committee of the Medical University of Guangdong. Informed and written consents were obtained from all participating subjects, which was in accordance with the Declaration of Helsinki.

\section{Questionnaires}

The COPD assessment test (CAT) consists of eight items with scores ranging from 0 to $5(0=$ no impairment, $5=$ greatest impairment). An overall score is calculated by adding the score from each item with total scores ranging from 0 to 40 . Higher scores indicated more severe health status impairment or a poorer COPD control [12].

\section{Collection and processing of induced sputum}

Sputum was induced as previously described [13]. Briefly, induced sputum from each subject was collected by means of inhalation of a nebulized 3\% saline solution over a 2-min period. Three volumes of dithiothreitol were added to sputum, and the sample was mixed vigorously on a shaker plate for 15 to $20 \mathrm{~min}$ in order to solubilize mucus. Cell counting was performed by an observer blind to the clinical characteristics of the subjects. A sample was considered adequate when the patient was able to expectorate at least $2 \mathrm{ml}$ of sputum, and slides contained $<10 \%$ squamous cells on differential cell counting. The percentage of sputum inflammatory cells was used for analysis. Sputum supernatants were kept at $-80{ }^{\circ} \mathrm{C}$ for further measurement of cytokines.

\section{Laboratory measurements}

Sputum HB-EGF levels were measured using commercially available enzyme-linked immunosorbent assay (ELISA) kits (Uscn Life Science, Inc., Wuhan, China). The minimum detection limit of the HB-EGF assay was $1.5 \mathrm{pg} / \mathrm{ml}$.

\section{Immunohistochemistry}

HB-EGF expression (Abcam, Cambridge, UK), N-cadherin and E-cadherin (Proteinthech, Chicago, USA), and vimentin (D21H3) (CST, Beverly, MA, USA) in lung tissues were investigated by immunocytochemical examination. Quantitative measurements of positive cells in the lung tissue were performed according to previously described methods [14]. Positive cells were expressed as a percentage of total cells. Peribronchial collagen deposition was detected by Masson trichrome staining. The area of peribronchial Masson trichrome staining (blue color) was visualized and 
was quantified by the software as a percentage of the total band area as previously described [15]. Sections were examined using a light microscope (BX51; Olympus, Japan) and quantified by computer algorithm using the Image Pro 6.1 software (Media Cybernetics).

\section{Immunofluorescence}

Immunofluorescent staining was performed on paraffinembedded lung tissue sections. The antibodies that were used in appropriate combinations were rabbit anti-human collagen I antibody (Proteinthech, Chicago, USA), rabbit anti-human collagen III antibody (Proteinthech, Chicago, USA), and mouse anti-human $\alpha$-SMA antibody (Abcam, Cambridge, UK).

\section{Western blotting assay}

Western blot analysis was used to detect changes in collagen types I and III, $\alpha$-smooth muscle actin (SMA), mitogenactivated protein kinase (ERK/MAPK), phosphorylated ERK (p-ERK), p38, phosphorylated (p-p38) (Cell Signaling Technology, USA), N-cadherin and E-cadherin (Proteinthech, Chicago, USA), and vimentin (D21H3) (CST, Beverly, MA, USA) as previously described [11].

\section{Cell viability assays, migration and proliferation}

To investigate HB-EGF's effects on tissue fibrosis, human lung fibroblasts were treated with different doses of recombinant human HB-EGF protein (R\&D Systems, Minneapolis, USA) for $48 \mathrm{~h}$. Cells were seeded into a 96well plate at a density of $1 \times 10^{4}$ cells/well. The cells were assayed with a cell counting kit (CCK)-8 (Liankebio, Hangzhou, China), which was used in order to determine cell proliferation according to the manufacturer's instructions. A 'scratch-wound' assay was used to assess fibroblast migration fibroblast as described previously [16].

\section{Statistical analysis}

Data were presented as the mean \pm SEM or median (IQR) unless otherwise stated. Statistical analyses were performed using the parametric two-tailed Student's $t$-test or nonparametric Mann-Whitney $U$-test for the comparison of two groups and ANOVA with post-hoc Tukey's corrections for the comparison of more than two groups. Spearman's rank correlation was used to test for dependence between two numeric variables. Statistical analysis was performed using GraphPad Prism 7.0 software (San Diego, CA, USA). $p<0.05$ was considered statistically significant.

\section{Results}

\section{Associations between sputum HB-EGF levels and clinical parameters}

HB-EGF levels were significantly higher in patients with COPD than those smokers without COPD and non-smokers
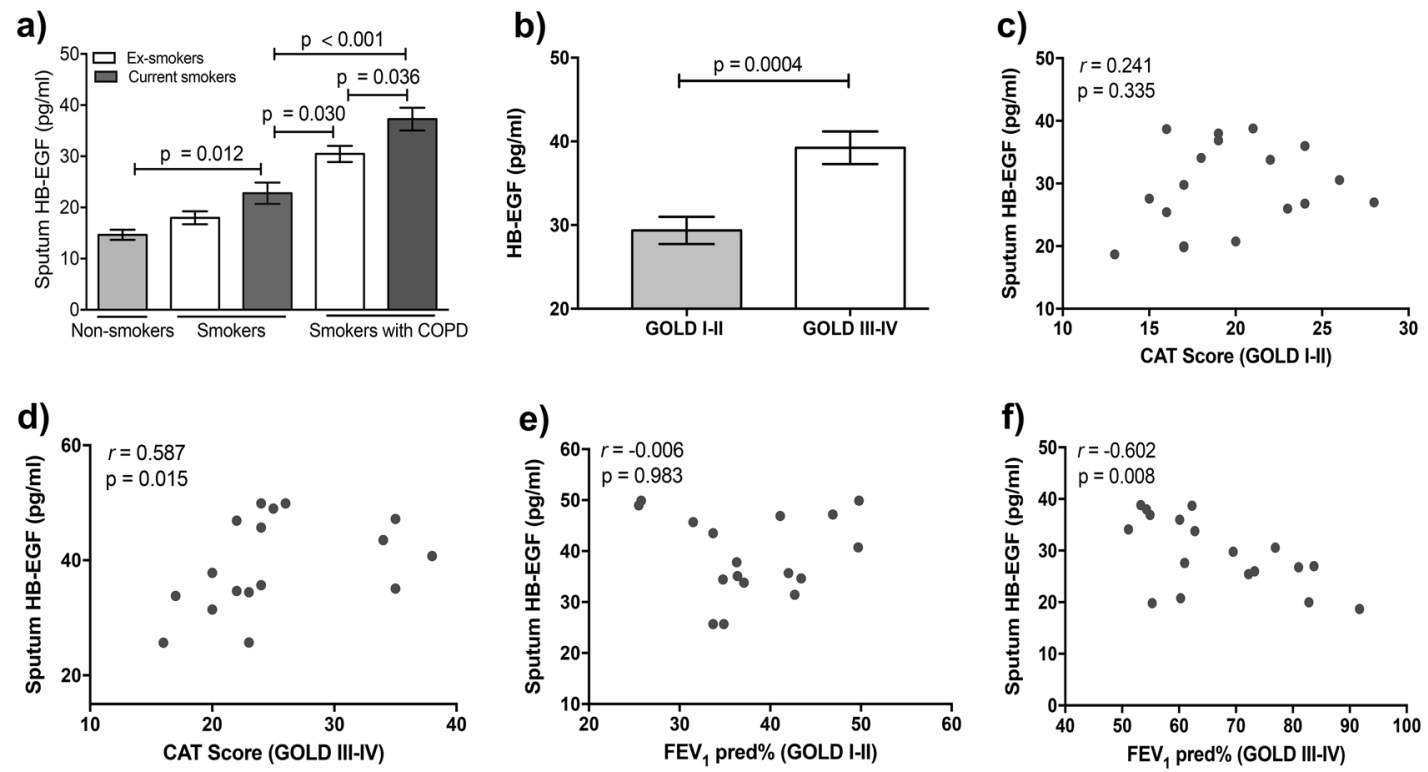

Fig. 1 HB-EGF expression in the sputum and correlations between sputum HB-EGF expression and disease severity. Sputum HB-EGF levels were quantified using ELISA $(\mathbf{a}, \mathbf{b})$. The correlations between the levels of HB-EGF in the sputum and COPD assess test $(\mathrm{CAT})$ scores, and FEV $_{1} \%$ Pred were investigated. The $r$ is the correlation coefficient using Spearman's test $(\mathbf{c}-\mathbf{f})$. Data are presented as mean \pm SEM 
Table 3 Comparison of COPD patients with HB-EGF levels above the mean $(34.2 \mathrm{pg} / \mathrm{ml})$ to patients with HB-EGF levels below the mean

\begin{tabular}{|c|c|c|c|}
\hline Variable & $\begin{array}{l}\text { HB-EGF levels } \\
<34.2 \mathrm{pg} / \mathrm{ml}(n= \\
17)\end{array}$ & $\begin{array}{l}\text { HB-EGF levels } \\
\geq 34.2 \mathrm{pg} / \mathrm{ml}(n= \\
18)\end{array}$ & $p$ value \\
\hline \multicolumn{4}{|l|}{ Sputum cell counts } \\
\hline Eosinophils, \% & $0.5(0-1.0)$ & $0(0-0.5)$ & 0.293 \\
\hline Neutrophils, \% & $39.5(34.0-42.0)$ & $50.3(40.3-61.6)$ & 0.003 \\
\hline Macrophages, \% & $59.5(56.5-65.0)$ & $48.5(38.0-58.6)$ & 0.009 \\
\hline Lymphocytes, \% & $0.5(0-1.0)$ & $0.25(0-1.0)$ & 0.521 \\
\hline \multicolumn{4}{|l|}{ Lung function } \\
\hline FVC, L & $3.03 \pm 0.2$ & $1.99 \pm 0.1$ & $<0.001$ \\
\hline $\mathrm{FEV}_{1}, \mathrm{~L}$ & $1.75 \pm 0.1$ & $1.03 \pm 0.1$ & $<0.001$ \\
\hline $\mathrm{FEV}_{1}, \%$ Pred & $62.9 \pm 4.4$ & $43.4 \pm 2.6$ & $<0.001$ \\
\hline $\mathrm{FEV}_{1} / \mathrm{FVC}, \%$ & $57.8 \pm 2.4$ & $50.9 \pm 1.8$ & 0.028 \\
\hline CAT scores & $19.4 \pm 0.9$ & $25.2 \pm 1.5$ & 0.003 \\
\hline $\begin{array}{l}\text { GOLD I-II/ } \\
\text { III-IV }, n\end{array}$ & $13 / 4$ & $5 / 13$ & 0.007 \\
\hline
\end{tabular}

Data are presented as mean \pm SEM or median (IQR), unless otherwise stated

$C A T$ COPD assess test, $F E V_{l}$ forced expiratory volume in $1 \mathrm{~s}, F V C$ forced vital capacity, GOLD Global Initiative for Chronic Obstructive Lung Disease

${ }^{a}$ COPD severity was graded following the GOLD

$(34.2 \pm 1.5 \mathrm{pg} / \mathrm{ml}$ vs. $20.4 \pm 1.3 \mathrm{pg} / \mathrm{ml}, p<0.01$ and $34.2 \pm$ $1.5 \mathrm{pg} / \mathrm{ml}$ vs. $12.4 \pm 1.0 \mathrm{pg} / \mathrm{ml}, p<0.001$, respectively). HBEGF expression in the sputum of current smokers with COPD was significantly higher than ex-smokers with COPD $(38.2 \pm 2.0 \mathrm{pg} / \mathrm{ml}$ vs. $28.4 \pm 1.7 \mathrm{pg} / \mathrm{ml}, p=0.036$; Fig. 1a). Moreover, patients with GOLD III/IV had significantly higher HB-EGF levels than patients with GOLD I/II $\quad(39.4 \pm 1.9 \mathrm{pg} / \mathrm{ml}$ vs. $29.3 \pm 1.6 \mathrm{pg} / \mathrm{ml}, \quad p=0.0004$; Fig. 1b). Although sputum HB-EGF levels were not associated with CAT scores or lung function ( $\mathrm{FEV}_{1} \%$ Pred) in patients with GOLD I/II (Fig. 1c, e), HB-EGF levels presented a significant positive association with CAT scores $(r$ $=0.587, p=0.015)$, but negative association with $\mathrm{FEV}_{1} \%$ Pred in patients with GOLD III/IV ( $r=-0.602, p=0.008$; Fig. 1d, f). When investigating all patients with COPD, those with HB-EGF levels above the mean $(34.2 \mathrm{pg} / \mathrm{ml})$ had higher sputum neutrophils and CAT scores, but lower lung function $\left(\mathrm{FEV}_{1} \%\right.$ Pred, $\mathrm{FEV}_{1} / \mathrm{FVC}$ ) compared to those with HB-EGF levels below the mean (Table 3). Collectively, these results suggest that sputum HB-EGF was increased in patients with COPD and may be associated with disease severity.

\section{HB-EGF expression is correlated with collagen deposition}

We next investigated HB-EGF expression and collagen deposition in lung tissues of subjects who had undergone surgery for lung tumors. We found that very few immunopositive cells were observed in the lung tissues from the control subjects, whereas there was a significant increase in HB-EGF staining density in COPD patients' airways when compared with smokers and non-smokers. HB-EGF expression in the lungs of current smokers with COPD was significantly higher than ex-smokers with COPD (40.6 $\pm 1.3 \%$ vs. $33.1 \pm 2.7 \%, p=0.026$; Fig. $2 \mathrm{a}$, b). Moreover, patients with GOLD III/IV had significantly higher HBEGF levels than patients with GOLD I/II $(41.3 \pm 1.4 \%$ vs. $34.0 \pm 2.1 \%, p=0.005$; Fig. 2c). Patients with COPD showed a tendency for an increase in collagen deposition in small airways when compared with smokers and nonsmokers (Fig. 2d). Patients with GOLD III/IV had significantly higher collagen deposition than patients with GOLD I/II $\quad(43.3 \pm 2.0 \%$ vs. $33.7 \pm 1.9 \%, \quad p=0.003$; Fig. 2e). Moreover, HB-EGF immunoreactivity positively correlated with collagen deposition in patients with GOLD III/IV ( $r=0.570 ; p=0.014$; Fig. 2g). However, similar results were not observed in patients with GOLD I/II ( $r=$ $0.228, p=0.474$; Fig. 2f).

\section{HB-EGF induced lung fibroblast activation}

The lung matrix is predominantly deposited by fibroblasts $[17,18]$. We next attempted to investigate whether HB-EGF exerted direct effects on lung fibroblasts in vitro. We found that exposure to recombinant human HB-EGF (rhHB-EGF) significantly upregulated lung fibroblast migration capacity and proliferation (Supplementary Fig. 1a, b). Treatment with rhHB-EGF also increased collagen I and collagen III production in fibroblasts (Fig. 3a-d).

The upregulation of $\alpha$-SMA expression is a characteristic marker for the fibroblast differentiation into activated fibroblasts (so-called myofibroblasts). Myofibroblasts have a role in the pathogenesis of airway remodeling in COPD [19]. Thus, we further detected $\alpha$-SMA expression in lung fibroblasts after treatment with rhHB-EGF using immunofluorescence and western blotting. We found that rhHBEGF significantly caused an increase in fibroblast $\alpha$-SMA protein expression (Fig. 3e, f). Together, these findings demonstrated that HB-EGF induced fibroblast activation and contributed to the pathogenesis of airway collagen deposition in COPD.

\section{Increased phosphorylation of p-38 and ERK in HB- EGF-induced collagen production}

The mitogen-activated protein kinase (ERK/MAPK) family is well known to play a key role in mediating inflammatory responses [11]. To assess whether ERK/MAPK pathways are involved in HB-EGF-induced collagen production, the effect of HB-EGF on activation of p38 and ERK was 
a)
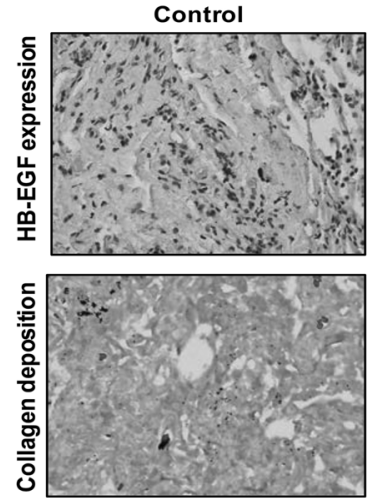

Smoker without COPD
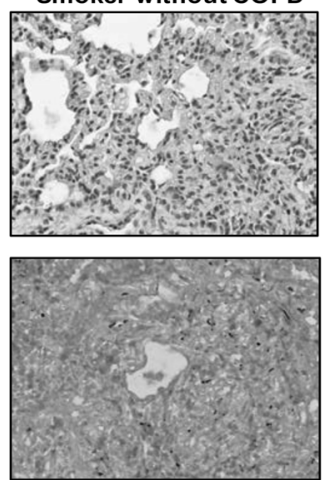
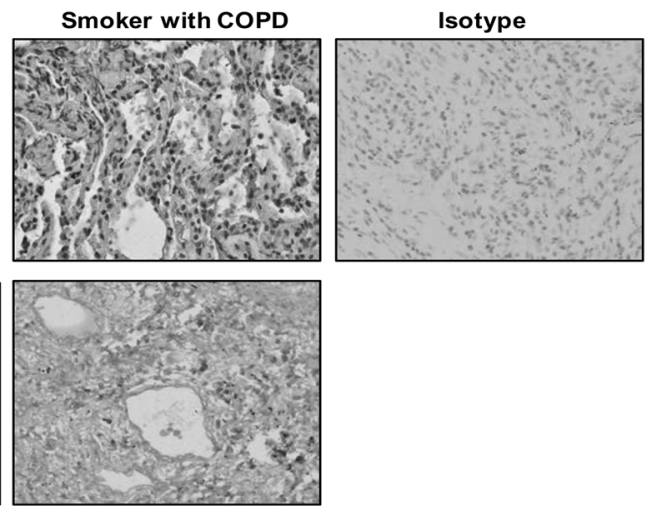
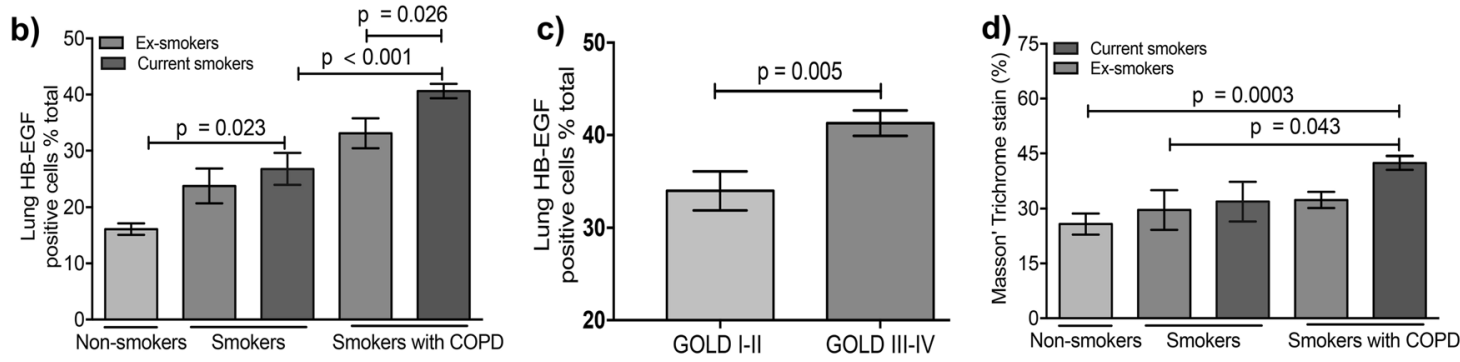

e)

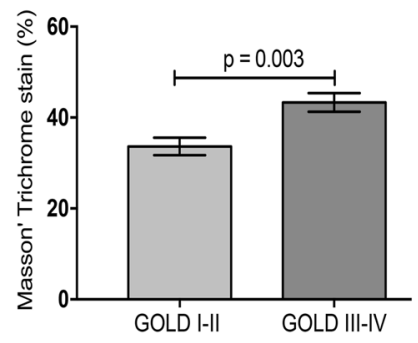

f)

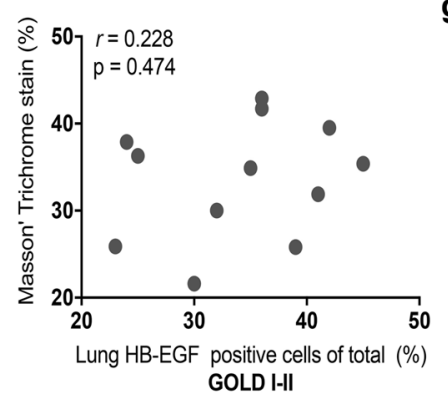

g)

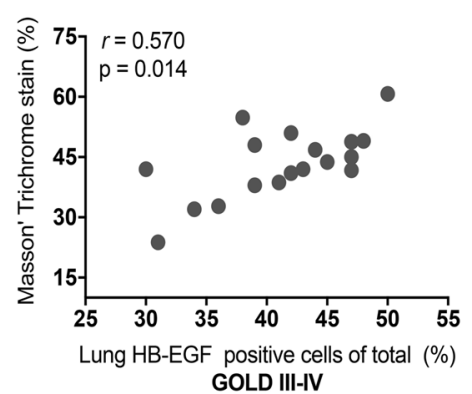

Fig. 2 HB-EGF expression and collagen deposition in the lung tissues of patients with COPD. The localization of HB-EGF in the lung tissues was investigated by immunostaining. Photomicrographs of blue staining showing collagen expression around the small airway walls. Representative photographs are shown (a). (all images are $\times 400$

evaluated by western blotting. As shown in Fig. 4, HB-EGF activated p38 and ERK phosphorylation in a timedependent and dose-dependent manner.

\section{HB-EGF expression in lung tissues and associated with airway EMT}

The percentages of $\mathrm{N}$-cadherin and vimentin positive cells had significantly increased in patients with COPD over that seen in smokers and non-smokers. (Fig. 5a, b; Fig. 6a, c). In contrast, E-cadherin expression had significantly decreased in patients with COPD over that in non-smokers (Fig. 5c; Fig. 6e). N-cadherin expression in GOLD IV was significantly higher than those in GOLD I (Fig. 6b). Although there was a trend to higher E-cadherin and vimentin expression in GOLD IV, there was no significant difference among these different groups (Fig. 6d, f). Moreover, these magnification). HB-EGF expression and collagen deposition in the lung tissues were quantified using the Image Pro 6.1 software (b-e). The relationship between HB-EGF expression and collagen deposition was assessed by Spearman's test $(\mathbf{f}, \mathbf{g})$. Data were presented as the mean $\pm \mathrm{SEM}$

mesenchymal markers significantly correlated with HBEGF expression in patients with GOLD III/IV (Fig. 6h, j, l), but similar results were not observed in patients with GOLD I/II (Fig. 6g, i, k).

\section{HB-EGF induces EMT-related gene expression in human airway epithelial cells}

Using immunofluorescence and western blotting, we detected $\mathrm{N}$-cadherin and vimentin expression in HBE cells after HB-EGF stimulation and found that it significantly increased $\mathrm{N}$-cadherin and vimentin expression in a concentration-dependent manner when compared with untreated control cells (Fig. 7a-d). In contrast, E-cadherin expression significantly decreased in HBE cells (Fig. 7e, f). Collectively, these data indicate that HB-EGF might induce EMT in HBE cells. 
a)

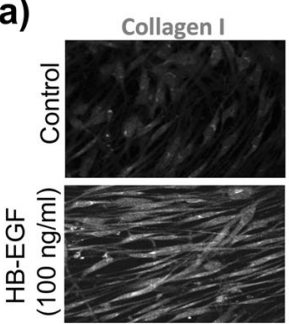

c)
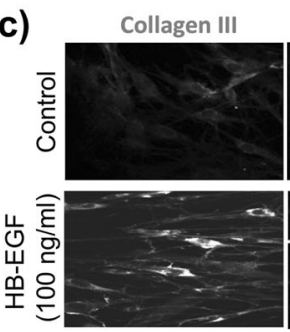

e)
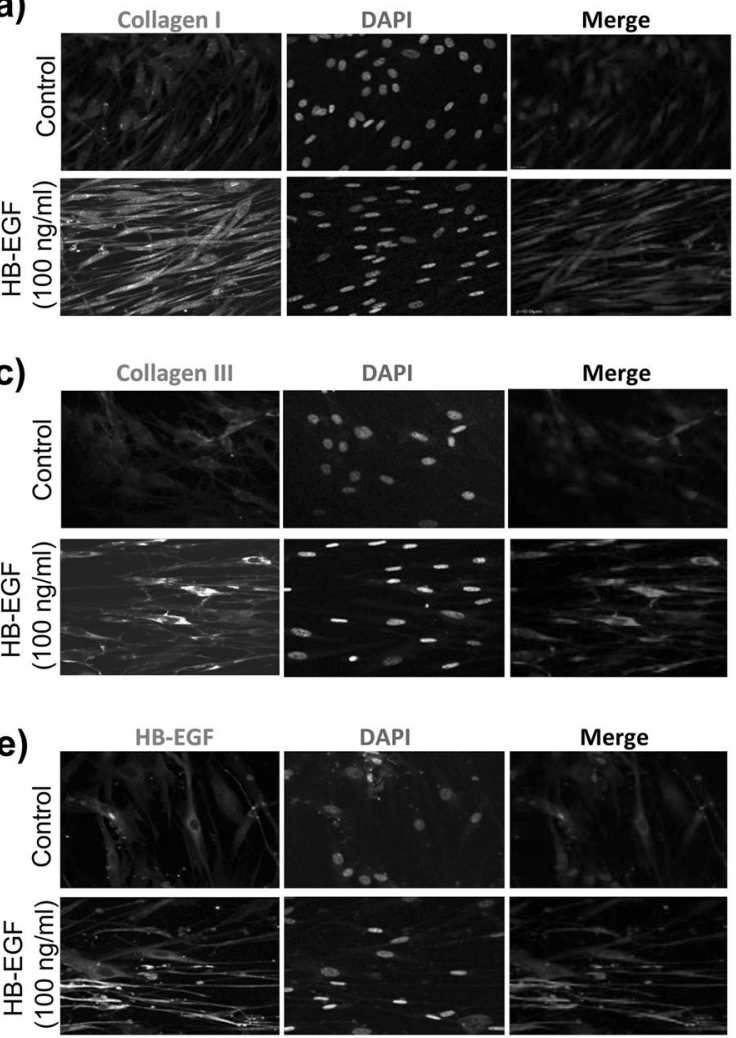

DAPI
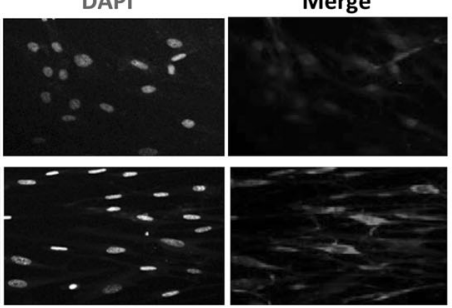

DAPI
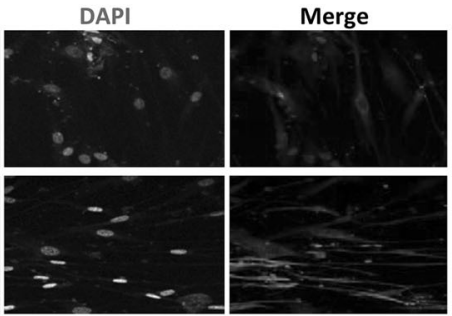

b)
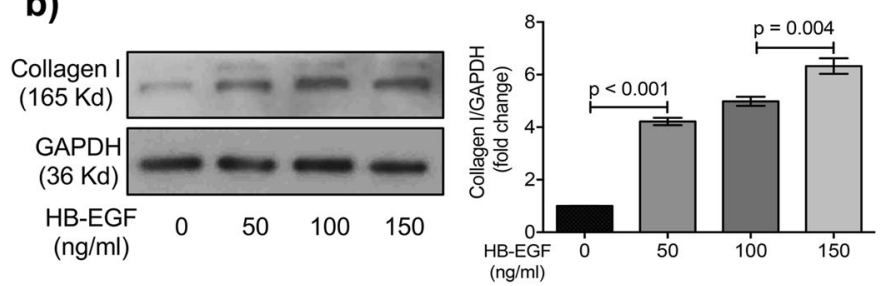

d)
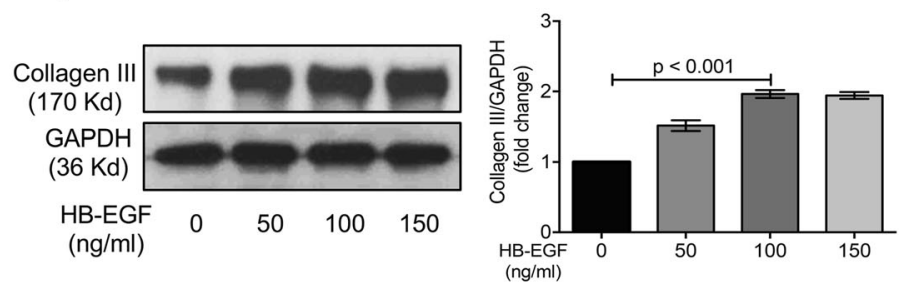

f)

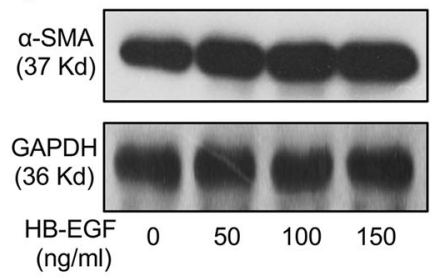

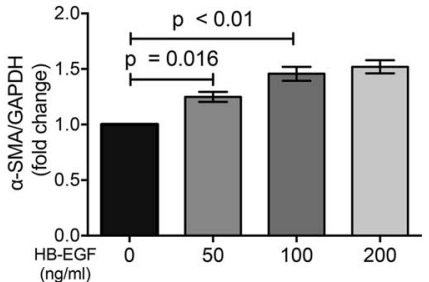

(ng/ml)

Fig. 3 The effect of HB-EGF treatment on human lung fibroblast cells. Collagen I, collagen III production and, $\alpha$-SMA expression upon stimulation by HB-EGF as determined by immunofluorescence staining

a)
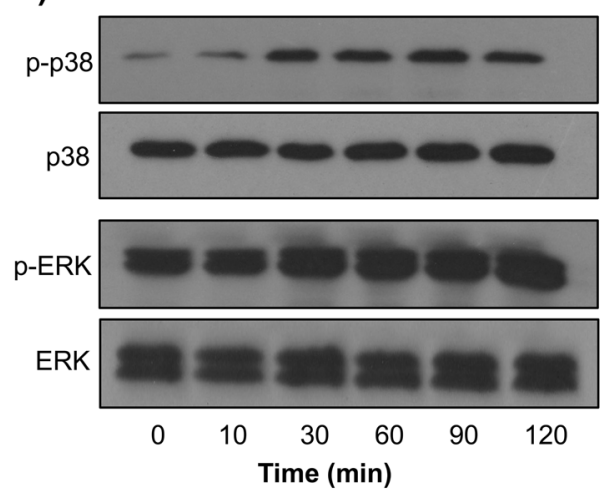

(a, c, e) and western blot analysis (b, d, f). Results were expressed as mean \pm SEM of three independent experiments

b)

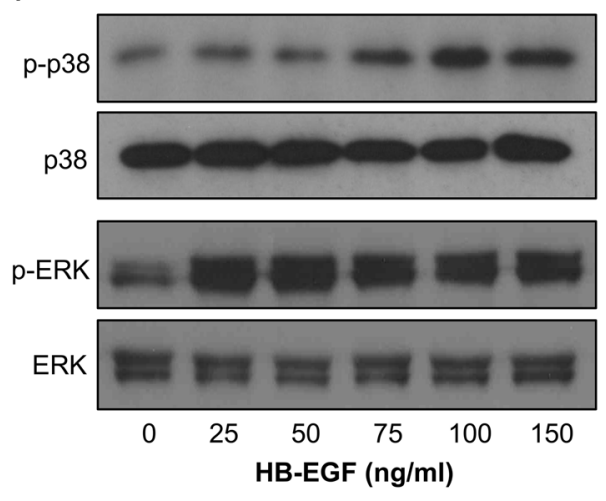

time-dependent manner (0, 10, 30, 60, 90, and $120 \mathrm{~min})$ (a); Recombinant human HB-EGF protein activated ERK and p38 phosphorylaton in a dose-dependent manner $(0,25,50,75,100$, and $150 \mathrm{ng} / \mathrm{ml}$ for 2 h) (b) lation of lung fibroblast cells with $100 \mathrm{ng} / \mathrm{ml}$ of recombinant human HB-EGF protein also induced phosphorylation of ERK and p38 in a

\section{Discussion}

In this study, we demonstrated that HB-EGF expression in sputum and lung tissues had significantly increased in patients with COPD compared to control subjects. HB-EGF expression was associated with disease severity, collagen

deposition, EMT-related markers ( $\mathrm{N}$-and E-cadherin and vimentin). In vitro, stimulation with HB-EGF promoted collagen production in lung fibroblasts and induced the EMT process in HBE cells. These results suggest that EGF blockade may be a treatment option for airway remodeling in COPD. 
a)
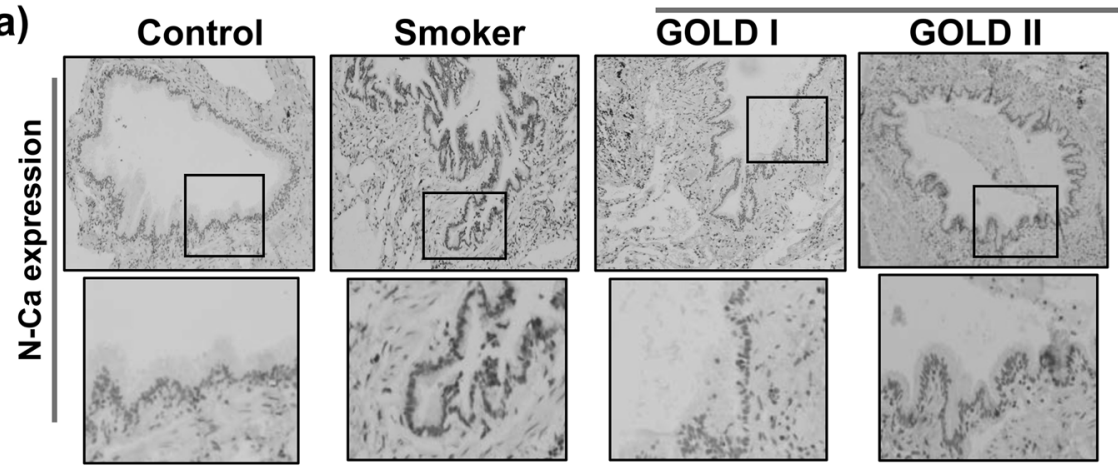

COPD
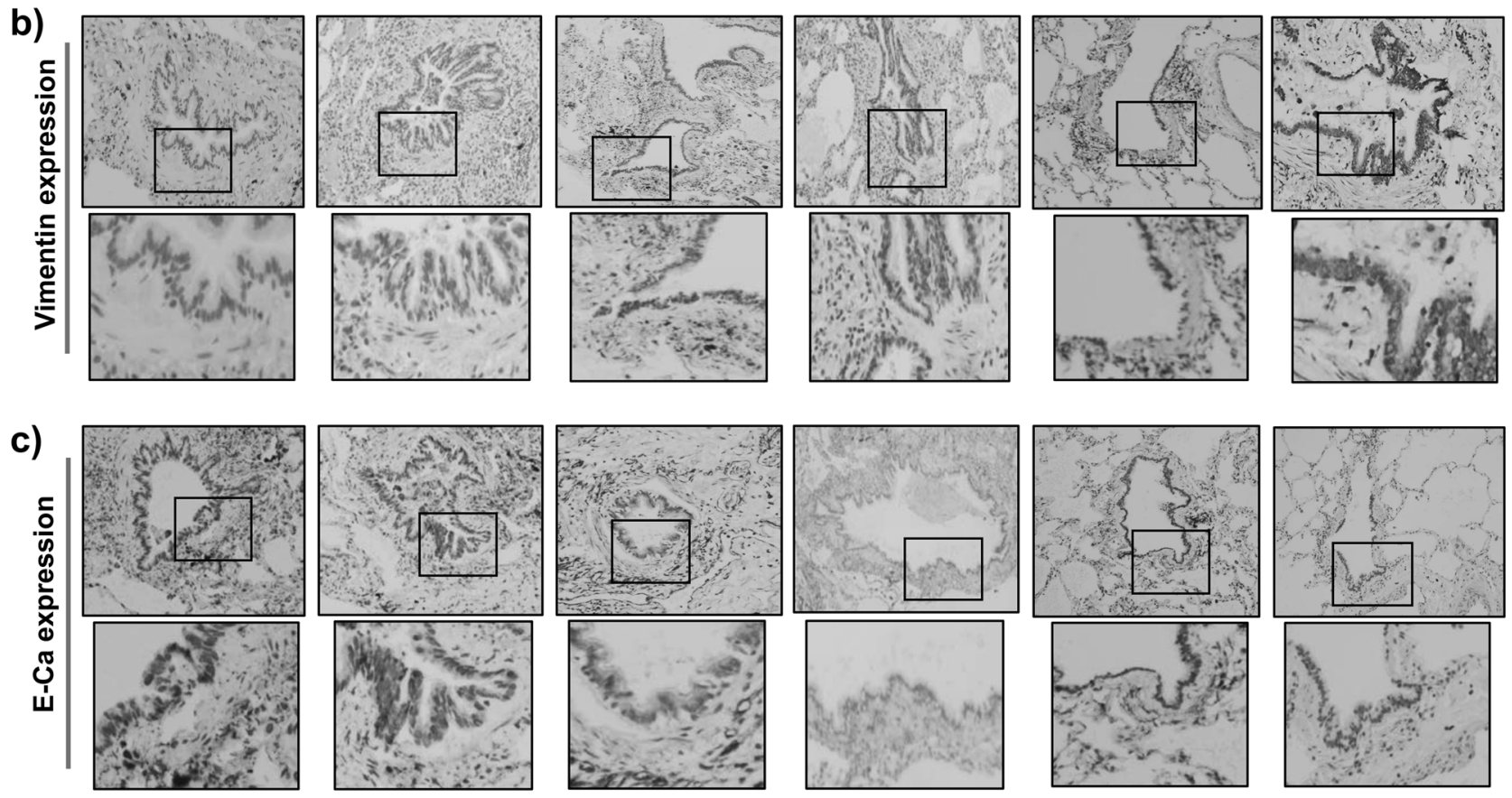

Fig. 5 The expression of EMT-related markers in the lung tissues of patients with COPD. N-cadherin, vimentin, and E-cadherin expression in the lung tissues were investigated using immunostaining. Representative photographs for control, smoker, and all the GOLD states are shown

COPD is accompanied by systemic inflammation and airflow limitations that occurs as a result of many mechanisms, particularly airway inflammation and smoking. HB-EGF, a member of the EGF superfamily, is expressed mainly in the airway epithelium [4]. In the present study, we found that HB-EGF expression in the sputum and lungs of current smokers with COPD was significantly higher than in the ex-smokers with COPD. Moreover, we observed that lung vimentin expression in current smokers with COPD was significantly higher than ex-smokers with COPD. These results indicated that smoking cessation may improve HB-EGF and vimentin expression in smokers with COPD. However, a large prospective study is needed to assess whether smoking-induced airway inflammation and structural changes are reversible after smoking cessation. Patients with GOLD III/IV had significantly higher HBEGF levels than patients with GOLD I/II. Moreover, patients with high levels of sputum HB-EGF had higher cytokine levels, CAT scores, and lower lung function compared to those with HB-EGF levels below the mean, thus revealing a cluster of clinical and pathophysiological variables associated with high HB-EGF levels. Together, these results strengthen the view that HB-EGF is implicated in COPD pathogenesis.

The remodeling process involves tissue repair and collagen deposition that contributes to lung function decline and airflow obstruction in COPD [12]. Collagens are synthetized primarily by fibroblasts as precursor molecules with the propeptides being cleaved during the process of secretion of the newly formed collagens [20]. However, it is unknown whether the increased HB-EGF levels participate in fibrosis onset in the small airways and are involved in airway remodeling pathogenesis in COPD. We examined collagen deposition in airways from patients with COPD 
a)

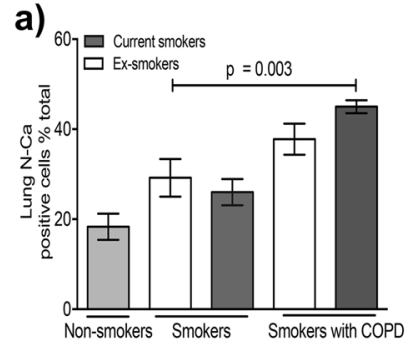

e)

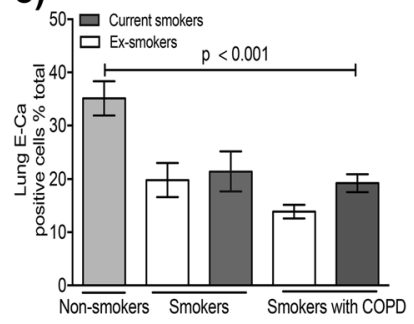

i)

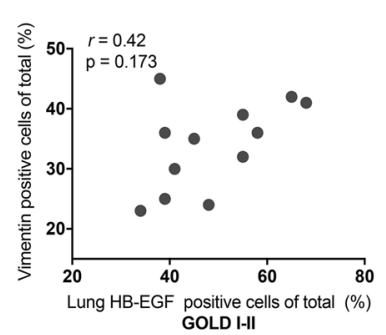

b)

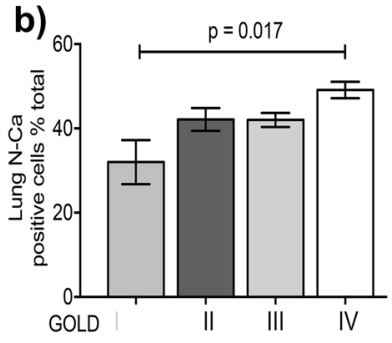

f)

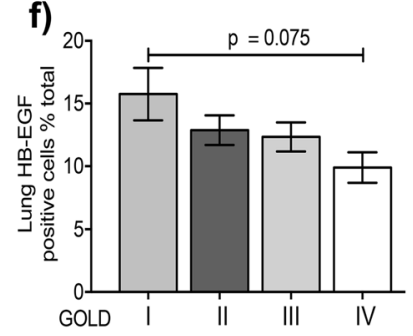

j)

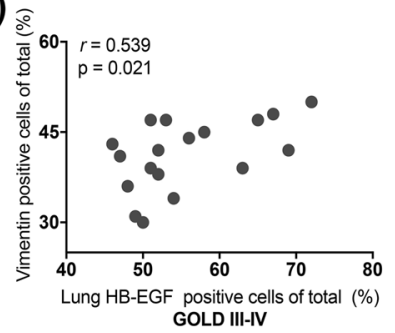

Fig. 6 Correlations between the expression of EMT-related markers in lung tissues and sputum HB-EGF levels. The expression of EMTrelated markers (N-cadherin, vimentin, E-cadherin) in lung tissues was

c)

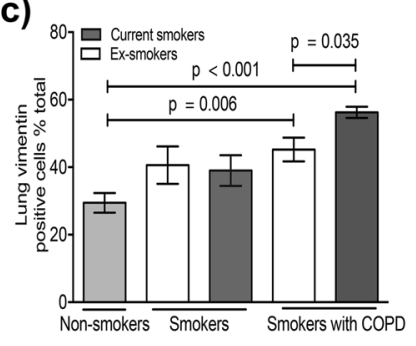

d)

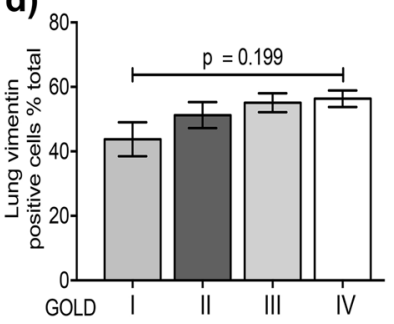

g)

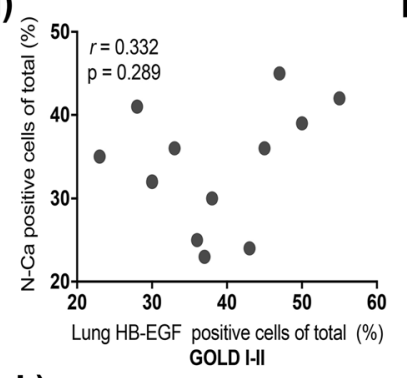

k)

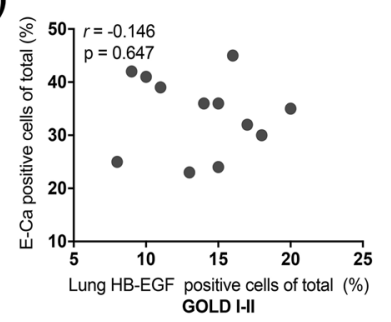

h)

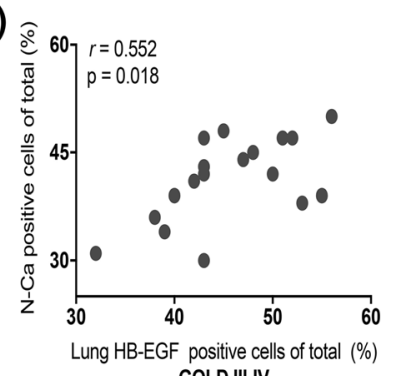

I)

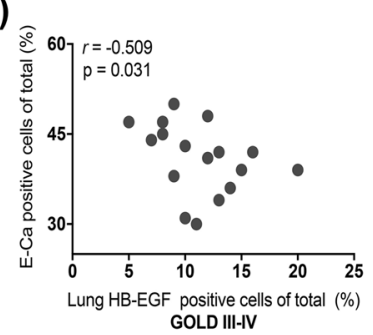

quantified using the Image Pro 6.1 software $(\mathbf{a}-\mathbf{f})$. The relationships between EMT-related markers and HB-EGF expression were assessed using Spearman's test. Data were presented as the mean \pm SEM

production in lung fibroblasts. Collectively, we presume that HB-EGF could activate lung fibroblasts and its downstream MAPK pathway and promote collagen production, which may enhance the airway remodeling process in COPD.

Airway epithelium represents the first line of defense against microorganisms and acts as a physical barrier between the host and the environment. Airway epithelium is composed of apical tight junctions and underlying adherent junctions [19]. It is now recognized that airway epithelium is involved in a variety of immunological mechanisms by releasing cytokines and interacting with immune cells [19]. EMT is a process essential in wound healing and tissue remodeling after injury [23]. Prevention of EMT is considered an effective measure for the inhibition of tissue remodeling. EMT was observed in several chronic inflammatory airway diseases such as asthma and COPD [19, 20, 23, 24]. Small airway fibrosis is the main contributor to airflow obstruction in COPD. EMT has been implicated in this process, and in large airways, it is associated with angiogenesis. Mahmood et al. showed that there was increased expression of EMT-related markers (EGFR, 
a)

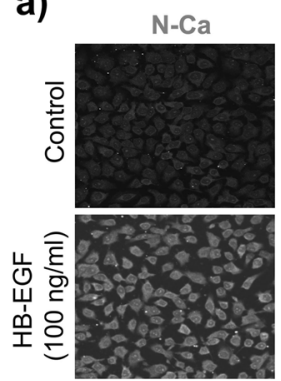

c)
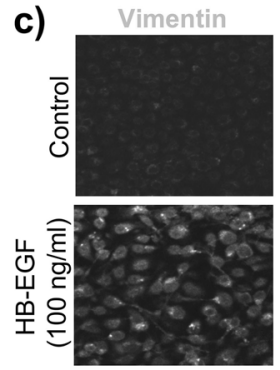

e)
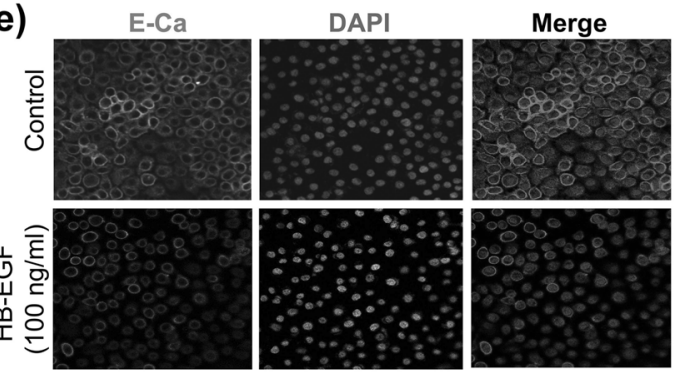

Fig. 7 HB-EGF promoted EMT production in HBE cells. HBE cells were treated with HB-EGF for $48 \mathrm{~h}$. Measurements of N-cadherin, Vimentin, and E-cadherin expression upon stimulation by HB-EGF as determined by immunofluorescence staining $(\mathbf{a}, \mathbf{c}, \mathbf{e})$, and western blot

vimentin, S100A4) in small airways of COPD compared to controls, but this was less than that in large airways [25]. Furthermore, they demonstrated that inhaled corticosteroids (ICS) has an anti-EMT effect in large airway samples in COPD [26]. Our study showed that the expression of $\mathrm{N}$ - and E-cadherin as a marker of EMT was associated with HB-EGF expression in COPD patients. In vitro, stimulation with HB-EGF promoted the EMT process in HBE cells. Collectively, these results suggest that EGF blockade may be a treatment option for airway remodeling in COPD.

There are some limitations to this study that need to be considered. First, a previous study showed that HB-EGF silencing with RNA interference inhibited cell cycle progression in lung cancer cells. HB-EGF was significantly and positively associated with primary lung tumors, and HBEGF expression predicted a poor survival outcome in patients [27]. Although resected normal tissues $>5 \mathrm{~cm}$ away from the tumor in our study, it should be noted that HBEGF influenced tumor growth and metastasis. Therefore,
$\mathrm{N}-\mathrm{Ca}$ $140 \mathrm{Kd}$

GAPDH $(36 \mathrm{Kd})$

HB-EGF (ng/ml)
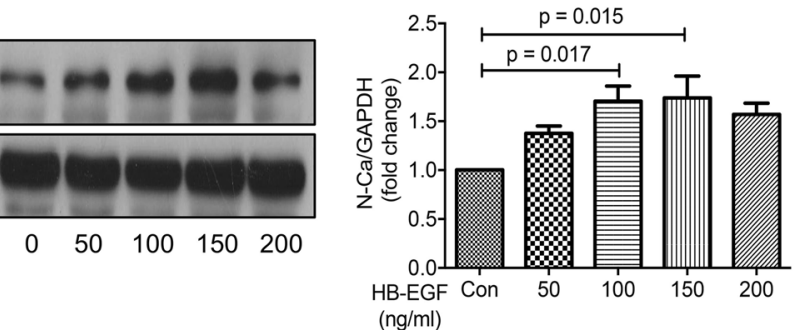

$(\mathrm{ng} / \mathrm{ml})$

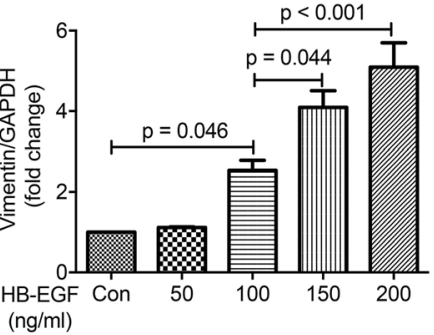

-Ca $125 \mathrm{Kd})$

GAPDH $(36 \mathrm{Kd})$

HB-EGF $(\mathrm{ng} / \mathrm{ml})$
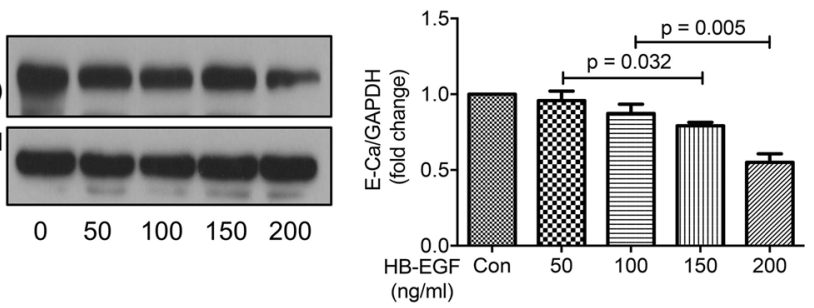

analysis (b, d, f). Results were expressed of three independent experiments. Data were presented as the mean \pm SEM of three independent experiments

HB-EGF expression in lung tissues should be interpreted cautiously due to this limitation. Second, a previous study showed that EMT-related protein expression was significantly high in the peripheral leading edge of non-smallcell lung cancer and related to tumor characteristics associated with poor prognosis [28]. EMT-related markers were also associated with clinicopathological parameters in adenocarcinomas and squamous cell lung carcinoma [29]. It should be noted that patients are not from a pure COPD population so lung cancer might have affected the results. Therefore, the expression of EMT-related markers in lung tissues should be interpreted cautiously due to this limitation.

In summary, we demonstrated that elevated HB-EGF expression is associated with disease severity in COPD patients. In addition, in vitro data showed that HB-EGF may promote airway remodeling in COPD by modulating airway fibrosis and pulmonary EMT. These findings suggest that HB-EGF may be a therapeutic target for intervention against airway remodeling in COPD. 
Acknowledgements Grant support: This work was supported by the Doctoral Startup Foundation of Affiliated Hospital of Guangdong Medical University (Tianwen Lai), the National Natural Science Foundation of China (81420108001), the National Natural Science Foundation of China (81700025), Medical and Health Science and Technology Project of Zhejiang (2018245859), and Beijing Medical Health Foundation (YWJKJJHKYJJ-HX32).

\section{Compliance with ethical standards}

Conflict of interest The authors declare that they have no conflict of interest.

\section{References}

1. Vestbo J, Hurd SS, Agustí AG, et al. Global strategy for the diagnosis, management, and prevention of chronic obstructive pulmonary disease. Am J Respir Crit Care Med. 2013;187:347-365.

2. Hogg JC, Chu F, Utokaparch S, et al. The nature of small-airway obstruction in chronic obstructive pulmonary disease. N Engl J Med. 2004;350:2645-2653.

3. Lai T, Tian B, Cao C, et al. HDAC2 Suppresses IL17A-Mediated Airway Remodeling in Human and Experimental Modeling of COPD. Chest 2017. pii: S001-3692(17)33044-1.

4. Raab G, Klagsbrun M. Heparin-binding EGF-like growth factor. Biochim Biophys Acta. 1997;1333:F179-F199.

5. Wang Q, Li H, Yao Y, et al. HB-EGF-Promoted Airway Smooth Muscle Cells and Their Progenitor Migration Contribute to Airway Smooth Muscle Remodeling in Asthmatic Mouse. J Immunol. 2016;196:2361-7.

6. Wang Q, Li H, Yao Y, et al. The overexpression of heparinbinding epidermal growth factor is responsible for Th17-induced airway remodeling in an experimental asthma model. J Immunol. 2010;185:834-41.

7. Hassan M, Jo T, Risse PA, et al. Airway smooth muscle remodeling is a dynamic process in severe long-standing asthma. $\mathbf{J}$ Allergy Clin Immunol. 2010;125:1037-.e3.

8. Gohy ST, Hupin C, Fregimilicka C, et al. Imprinting of the COPD airway epithelium for dedifferentiation and mesenchymal transition. Eur Respir J. 2015;45:1258-72.

9. Kalluri R, Weinberg RA. The basics of epithelial-mesenchymal transition. J Clin Invest. 2009;119:1420-8.

10. Bartis D, Mise N, Mahida RY, et al. Epithelial-mesenchymal transition in lung development and disease: does it exist and is it important? Thorax. 2014;69:760-5.

11. Lai $\mathrm{T}, \mathrm{Wu} \mathrm{D}, \mathrm{Chen} \mathrm{M}$, et al. YKL-40 expression in chronic obstructive pulmonary disease: relation to acute exacerbations and airway remodeling. Respir Res. 2016;17:31. 24

12. GOLD Committee. Global strategy for the diagnosis, management, and prevention of COPD. http://www.goldcopd.org/ guidelines-pocket-guide-to-copd -diagnosis.html. Accessed 11 Jan 2016.

13. Kips JC, Fahy JV, Hargreave FE, et al. Methods for sputum induction and analysis of induced sputum: a method for assessing airway inflammation in asthma. Eur Respir J Suppl. 1998;26:9e12S.

14. Xanthou G, Alissafi T, Semitekolou M, et al. Osteopontin has a crucial role in allergic airway disease through regulation of dendritic cell subsets. Nat Med. 2007;13:570-8.

15. Fattouh R, Midence NG, Arias K, et al. Transforming growth factor-beta regulates house dust mite-induced allergic airway inflammation but not airway remodeling. Am J Respir Crit Care Med. 2008;177:593-603.

16. Liang CC, Park AY, Guan JL. In vitro scratch assay: a convenient and inexpensive method for analysis of cell migration in vitro. Nat Protoc. 2007;2:329-33.

17. Krimmer DI, Burgess JK, Wooi TK, et al. Matrix proteins from smoke-exposed fibroblasts are pro-proliferative. Am J Respir Cell Mol Biol. 2012;46:34-39.

18. Kranenburg AR, Willems-Widyastuti A, Moori WJ, et al. Enhanced bronchial expression of extracellular matrix proteins in chronic obstructive pulmonary disease. Am J Clin Pathol. 2006;126:725-35.

19. Park IH, Kang JH, Shin JM, et al. Trichostatin A Inhibits Epithelial Mesenchymal Transition Induced by TGF- $\beta 1$ in Airway Epithelium. PLoS ONE. 2016;11:e0162058.

20. Weber CE, Li NY, Wai PY, et al. Epithelial-mesenchymal transition, TGF-beta, and osteopontin in wound healing and tissue remodeling after injury. J Burn Care Res. 2012;33:311-8.

21. Harju T, Kinnula VL, Pääkkö P, et al. Variability in the precursor proteins of collagen I and III in different stages of COPD. Respir Res. 2010;30:165.

22. Sun C, Zhu M, Yang Z, et al. LL-37 secreted by epithelium promotes fibroblast collagen production: a potential mechanism of small airway remodeling in chronic obstructive pulmonary disease. Lab Invest. 2014;94:991-1002.

23. Georas SN, Rezaee F. Epithelial barrier function: at the front line of asthma immunology and allergic airway inflammation. J Allergy Clin Immunol. 2014;134:509-20.

24. Milara J, Peiro T, Serrano A, et al. Epithelial to mesenchymal transition is increased in patients with COPD and induced by cigarette smoke. Thorax. 2013;68:410-20.

25. Mahmood MQ, Sohal SS, Shukla SD, et al. Epithelial mesenchymal transition in smokers: large versus small airways and relation to airflow obstruction. Int J Chron Obstruct Pulmon Dis. 2015;10:1515-24.

26. Sohal SS, Soltani A, Reid D, et al. A randomized controlled trial of inhaled corticosteroids (ICS) on markers of epithelialmesenchymal transition (EMT) in large airway samples in COPD: an exploratory proof of concept study. Int J Chron Obstruct Pulmon Dis. 2014;9:533-42.

27. Hsieh $\mathrm{CH}$, Chou YT, Kuo $\mathrm{MH}$, et al. A targetable HB-EGFCITED4 axis controls oncogenesis in lung cancer. Oncogene. 2017;36:2946-56.

28. Mahmood MQ, Ward C, Muller HK, et al. Epithelial mesenchymal transition (EMT) and non-small cell lung cancer (NSCLC): a mutual association with airway disease. Med Oncol. 2017;34:45.

29. Kim SH, Kim JM, Shin MH, et al. Correlation of epithelialmesenchymal transition markers with clinicopathologic parameters in adenocarcinomas and squamous cell carcinoma of the lung. Histol Histopathol. 2012;27:581-91. 